\title{
A genomic view of eukaryotic DNA replication
}

\author{
David M. MacAlpine \& Stephen P. Bell* \\ Howard Hughes Medical Institute, Department of Biology, Massachusetts Institute of Technology, \\ Cambridge MA 02139, USA; Tel: +1(617)253-2054; Fax: +1(617)253-4043; E-mail: spbell@mit.edu \\ * Correspondence
}

Key words: genomics, microarray, origin recognition complex (ORC), origin selection, replication timing

\begin{abstract}
Recent advances in DNA microarray technology have enabled eukaryotic replication to be studied at whole-chromosome and genome-wide levels. These studies have provided new insights into the mechanisms that influence origin selection and the temporally co-ordinated activation of replication initiation from these sites. Here we describe multiple microarray-based approaches that have been used to study DNA replication in both $S$. cerevisiae and higher eukaryotes. We have also compiled the data from the yeast microarray-based replication studies to generate a comprehensive list of origins that has been verified in three independent studies. The comprehensive nature of the microarray-based studies has revealed clear connections between chromosome organization and the pattern of replication. For example, in yeast, the centromeric proximal sequences are consistently early replicating and telomeric regions are consistently late replicating. The metazoan studies reveal a recurring theme of gene-dense transcriptionally active regions of the genome replicating before gene-sparse regions. In addition to the insights they have provided already, microarray-based replication assays combined with genetic analysis will provide a powerful new approach to define the mechanisms that regulate replication origin function.
\end{abstract}

\section{Introduction}

Each time a eukaryotic cell divides, a perfect copy of the genome must be generated within the confines of S-phase. In metazoan organisms, this regulation is dynamic, responding to changes in developmental programme and the length of Sphase. There are two primary control points that regulate the pattern of chromosomal replication: selection of potential origins of replication and the activation of a subset of these sites during $\mathrm{S}$ phase. Although we have an increasing understanding of the proteins involved in the initiation of eukaryotic DNA replication, the specific chromosomal elements that direct the initiation of DNA replication and the chromosomal features that control the activity of each origin of replication are not fully understood.

Origin selection is mediated by the formation of a multiprotein complex termed the prereplicative (pre-RC) complex. Assembly of the pre-RC is initiated by the binding of the origin recognition complex (ORC) to origin-proximal DNA sequences (reviewed in Bell 2002, Mendez \& Stillman 2003). In $S$. cerevisiae, ORC exhibits in-vitro sequence specificity for the ARS consensus sequence (ACS) found at each origin. During the G1 phase of the cell cycle, ORC uses the energy of ATP hydrolysis to recruit and assemble additional proteins into a fully formed pre-RC (Bowers et al. 2004). These additional proteins include the pre- $\mathrm{RC}$ assembly factors Cdc6 and Cdt1 and the putative replicative 
DNA helicase, the Mcm2-7 complex. All the proteins required for pre-RC formation are conserved in higher eukaryotes, yet comparatively less is know about the DNA sequences or chromosome features that define the sites of their assembly (reviewed in Gilbert 2004). No clear consensus sequence has emerged from the characterised metazoan origins, and ORC from higher eukaryotes exhibits little sequence specificity in vitro (Vashee et al. 2003, Remus et al. 2004). Although the formation of a pre- $\mathrm{RC}$ is a prerequisite for initiation of replication, not all sites of pre- $\mathrm{RC}$ formation are equivalent and only a subset of these complexes will direct replication initiation in the subsequent S-phase (Wyrick et al. 2001, Raghuraman et al. 2001).

For a given cell type under a given growth condition, each part of the genome replicates at a characteristic time within $\mathrm{S}$ phase. Some regions of the genome are consistently replicated early in S-phase, some in the middle and still others at the end (Goldman et al. 1984, Taljanidisz et al. 1989, Friedman et al. 1997). This property is a conserved feature of eukaryotic genomes, although the functional importance of this type of replication control remains unclear (reviewed in Gilbert 2002). The time of origin activation across the genome is a primary determinant of this temporal pattern of replication.

The time each origin is activated during $\mathrm{S}$ phase is related to its chromosomal position. Early studies of metazoan replication noted that heterochromatic regions of chromosomes were consistently replicated later than their euchromatic counterparts (Stambrook \& Flickinger 1970). The minimal DNA sequences required to direct replication initiation are referred to as the replicator (reviewed in Bell et al. 1993). Studies in S. cerevisiae strongly suggest that the replicator itself is not sufficient to dictate the temporal program of initiation (Friedman et al. 1996). For example, if a replicator that normally initiates early is moved to a latereplicating region of the chromosome, it takes on the late-replicating nature of its surrounding sequences. The mechanisms that control replication timing are still poorly understood but appear to involve effects of chromatin structure (Vogelauer et al. 2002, Aparicio et al. 2004) and control of the activity of crucial activating kinases (Shirahige et al. 1998, Donaldson et al. 1998, Santocanale \& Diffley 1998).
Until recently, our understanding of how replication is regulated was derived from experiments analysing a small subset of the replication origins of a given genome. These approaches greatly increased our understanding of how individual origins are selected in $S$. cerevisiae and, to a lesser extent, in metazoan organisms. A weakness of these studies is that they are unable to look at replication of chromosomes in a comprehensive manner necessary to understand how origins of replication are regulated. The recent development of DNA microarray-based approaches to characterize replication dynamics at the genome-wide level represents a powerful new method to understand the selection and control of origins of replication. This review focuses on the different microarray-based methodologies that have been used to address eukaryotic DNA replication and their impact.

\section{Identification and characterisation of yeast origins of replication}

Origin selection and regulation is best understood for the yeast $S$. cerevisiae (Mendez \& Stillman 2003). S. cerevisiae origins of replication were initially identified by a screen for autonomous replicating sequences (ARS) that allowed a plasmid to be stably maintained in the yeast nucleus (Hsiao \& Carbon 1979, Stinchcomb et al. 1979). Subsequent studies using two-dimensional gel electrophoresis demonstrated that a subset of these sequences acted as origins of replication both in plasmids (Brewer \& Fangman 1987) and in their native chromosomal context (Brewer \& Fangman 1991). Combining these assays with mutagenesis of origin-proximal DNA defined the minimal replicator as a region of $100-150 \mathrm{bp}$ that included three to four 10-12 bp DNA sequence elements that were required for origin function (reviewed in Newlon \& Theis 1993, Bell 1995). Interestingly, the DNA sequence of only one of these elements, referred to as the ARS consensus sequence (ACS) is conserved between different origins (Rao et al. 1994).

The first efforts to identify origins across an entire chromosome used two-dimensional gel electrophoresis to survey the smallest yeast chromosomes for origin activity (Reynolds et al. 1989, Newlon et al. 1993, Friedman et al. 1997). Despite 
these Herculean efforts, the total number of origins identified by these methods was less than 30 and the vast majority of the yeast genome remained uncharacterized. To fully catalogue and characterize the replication of a genome, systematic methods were developed to comprehensively identify origins of replication. Here we describe three microarray-based approaches to identify these regions and discuss the advantages and disadvantages of each technique. We have also compared the different data sets to generate a comprehensive list of potential yeast origins of replication identified by all three methods. Finally, we discuss the new insights into yeast replication and chromosome biology that these techniques have provided.

\section{Identification of replication origins using replication timing profiles: a density transfer approach}

The first genome-wide look at $S$. cerevisiae replication used a modern twist on the classic Messelson and Stahl experiment demonstrating the semi-conservative replication of DNA (Meselson \& Stahl 1958). Heavy isotope labelling in combination with Affymetrix microarray technology was used to characterize the temporal pattern of replication across the entire $S$. cerevisiae genome (Raghuraman et al. 2001). After extended growth in media with ${ }^{13} \mathrm{C}$ and ${ }^{15} \mathrm{~N}$ precursors to fully label genomic DNA with 'heavy' isotopes, cells were arrested at the $\mathrm{G} 1 / \mathrm{S}$ transition of the cells cycle. These cells were then allowed to proceed synchronously into S-phase in the presence of isotopically light media and cells were collected during eight time intervals that spanned $\mathrm{S}$ phase. The newly synthesized DNA incorporated the 'light' isotopes resulting in hybrid 'heavy: light' DNA molecules. After cutting, the replicated and nonreplicated DNA were separated and purified by ultracentrifugation.

The ability to separate replicated from nonreplicated DNA allowed the authors to generate a replication timing profile of the yeast genome. Samples of the eight purified replicated and nonreplicated DNA were fluorescently labelled and individually hybridised to Affymetrix arrays that contained oligonucleotides representing the majority of the yeast open reading frames. For every locus on the array, the replicated (heavy: light) hybridization signal for all time points was summed (a measure of replicated DNA) and divided by the sum of the replicated and non-replicated signals for all eight time points (a measure of total DNA) to determine a heavy : light ratio. The resulting ratio is directly related to the time of replication for each point on the array. Sequences that replicate early in S-phase will have a high heavy : light signal across the majority of time points and therefore an elevated heavy: light ratio. In contrast, sequences that replicate later in $\mathrm{S}$ phase will have a significant heavy: light signal only at later time points and therefore a lower total heavy: light signal and a correspondingly low heavy : light ratio.

To identify origins of replication, a replication timing profile was generated for each chromosome by plotting the heavy: light ratio as a function of chromosomal position. To reduce the inherent noise in these data, smoothing algorithms were developed that took into account the fact that adjacent sequences should have related times of replication. Assuming that each origin generates a bidirectional pair of replication forks, any region of the genome that replicates earlier than its neighbouring sequences on either side must contain an origin of replication. Thus, the peaks of the replication timing profile represent sites of the earliest local replication and can be used to localize origins of replication along the chromosome. The relative height of the peak at an origin is a function of its temporal pattern of activation. The origins that initiate earliest will generally be associated with the highest peaks (however, see below). By identifying the peaks in the replication timing profiles for each chromosome, 332 distinct origins of replication were identified in this study. Many of these sites overlapped with previously characterized origins of replication. For example, of 18 known efficient replication origins, 13 of these were located within $5 \mathrm{~kb}$ of peaks defined by the replication timing profiles.

\section{Identification of replication origins using replication timing profiles: a copy number approach}

An alternative approach used to identify $S$. cerevisiae origins exploited the 2-fold increase in copy 
number of replicated versus non-replicated sequences (Yabuki et al. 2002). The ability to use DNA microarrays to detect the different quantity of DNA between replicated and non-replicated regions of the genome was first demonstrated for E. coli DNA replication (Khodursky et al. 2000). As in the density transfer studies above, this approach involved collecting DNA samples at 22 intervals from cells passing synchronously through $\mathrm{S}$ phase. These samples were labelled and hybridized to an Affymetrix microarray (without separating DNA into replicated and non-replicated samples). In this case, instead of determining a 'heavy : light ratio' for each locus, the authors simply summed the total amount of hybridized DNA observed for the multiple time intervals. Sites that replicated early would have a higher summed signal (due to having spent more time with 2-fold more DNA) and those that replicated later would have a lower summed value. Replication profiles were generated using a simple moving average of the data. Again, locally early-replicating regions were considered to be origins of replication and the 247 identified peaks could be classified into two distinct groups of early and late replicating origins. Although fewer origins of replication were identified by this method, the replication timing profiles generated by examining copy number were similar to those obtained using the density transfer method (see below).

A modification of the copy number protocol allowed the exclusive mapping of origins activated early in $\mathrm{S}$ phase. In these experiments, hydroxyurea (HU) was added to cells prior to release into $\mathrm{S}$ phase. The addition of $\mathrm{HU}$ has two effects (reviewed in Pasero et al. 2003): (1) any replication forks emanating from an origin will stall within $10-15 \mathrm{~kb}$ of the origin due to low dNTP levels (HU is an inhibitor of dNTP synthesis); (2) the resulting stalled forks induce the intra-S phase checkpoint which prevents initiation of replication from late origins of replication (Shirahige et al. 1998, Santocanale \& Diffley 1998). Thus, when cells were treated with high concentrations of HU, only sequence proximal to early origins of replication exhibited an increased copy number. Using this approach, 116 of the 122 origins identified as early activating through analysis of the copy number replication profile were found to initiate in the presence of HU.

\author{
Identification of potential origins of replication \\ by mapping sites of pre-RC formation: \\ a genome-wide location analysis \\ (ChIP-Chip) approach
}

In complementary work, genome wide-location analysis (Ren et al. 2000) was used to identify the binding sites of the pre-RC components, ORC and the Mcm2-7 complex, across the yeast genome (Wyrick et al. 2001). To identify the binding sites of these proteins, chromatin immunoprecipitation was combined with a custom PCR fragment-based microarray representing all of the yeast open reading frames (ORFs) and intergenic sequences. Briefly, crosslinked, sheared chromatin was immunoprecipitated with antibodies to the ORC and the Mcm2-7 complex. The coimmunoprecipated DNA was fluorescently labelled and hybridized to the microarray. As a control, differentially labelled non-enriched DNA was simultaneously hybridized to the array. For each sequence on the array, the ratio of immunoprecipitated DNA hybridization (enriched) signal and the control non-enriched signal was measured. Sequences that interact with the target protein will have a higher ratio of enriched to non-enriched signal. Greater than 420 sites at which both ORC and MCM proteins were localized, termed pro-ARSs, were identified. As ORC and MCM are both essential components for pre-RC assembly, the identified sites each represented potential origins of replication.

\section{Advantages and disadvantages of array-based origin identification}

As methods to identify origins of replication, each of these genomic techniques has advantages and disadvantages. Both of the methods used to generate replication timing profiles have the advantage of assessing DNA replication directly. The replication timing profiles generated by the density transfer method offers the most direct measurement of DNA replication. Only those sequences that have been replicated will appear in the heavy: light fraction. In contrast, copy number studies do not directly assess replication but assume that any increase in abundance is due to replication. Although the studies in yeast suggest 
that this is a good assumption, it is possible that the increased complexity and/or increased repetitive sequences of metazoan organisms could make this approach more difficult. A major advantage of both of these approaches is that the replication profiles generated provide information not only about the location of origins but also the temporal pattern of activation. In addition, these profiles have the potential to reveal information about replication fork rates.

A weakness of the replication profile studies is that there are several differences in origin function that can be responsible for a change in the replication profile. Both time and efficiency of origin activation will contribute to the overall shape of the replication profile. Although efficient, early activating origins will always be represented by the highest peaks in these replication profiles, it is impossible to distinguish an efficient late-replicating origin from an inefficient early-replicating origin. Both of these circumstances result in a reduced peak height. This is because, when an origin does not initiate (as would occur in cell cycles during which an inefficient origin did not initiate), that origin DNA must be replicated later by replication forks that emanate from an adjacent origin. Thus an early inefficient origin will have a peak height that will be similar to that of an efficient late-replicating origin. The reduction in peak height associated with inefficient origins may also prevent their identification.

Several features of these early replication timing profile studies limited their resolution. As with all microarray-based methods, the density of the DNA probes provides an upper limit of resolution. In addition, for the replication profile studies, the degree of cell-cycle synchrony, the extent of data smoothing, and the proprietary nature of the Affymetrix arrays (which prevented a precise knowledge of the sequences represented on the array) all contributed to the resolution of these studies being significantly lower than the density of the DNA probes. Currently, the resolution of these studies is likely to be in the range of a few kilobases, which prevents a definitive identification of the DNA sequences most important for origin function.

As published, both techniques for generating replication timing profiles were laborious and time consuming but recent studies suggest that they can be simplified. The requirement to hybridize each
S-phase time interval to separate microarrays was particularly onerous. Interestingly, studies have demonstrated that similar results can be achieved by pooling the samples from all of the S-phase time intervals and hybridizing to a single array (Raghuraman et al. 2001). Similarly, more recent studies indicate that the same results can be obtained with the copy number method on oligobased arrays using simultaneous hybridization of differentially labelled control unreplicated DNA and pooled S-phase DNA samples (Blitzblau \& Bell, in preparation).

By reducing the number of origins that initiate and the extent of fork migration, the use of arresting concentrations of HU could provide additional simplicity to origin identification. By allowing only sequences immediately adjacent to origins to replicate, the addition of $\mathrm{HU}$ can provide a more 'binary' output. However, it is important to keep in mind that only early origins of replication can be monitored in this manner in a wild-type cell and that altered dNTP pools may influence the density and activity of specific origins (Anglana et al. 2003). It is possible that inactivation of the intraS-phase checkpoint could allow the identification of later-replicating origins but it would be important to keep in mind the possibility that this type of mutation could also influence origin use in other ways. The use of HU and microarrays to classify early- and late-activating origins of replication could also be a powerful tool to characterize mutants that affect replication timing. Specifically, this method could provide a sensitive assay for mutations that abrogate the intra-S-phase checkpoint (see summary) or that allow late-activating origins to initiate early.

The primary advantage of origin mapping based on genome-wide location analysis is the ability to map potential origins at higher resolution. Because the analysis of this data does not involve the use of pools of cells synchronously passing through $\mathrm{S}$ phases, moving averages or other types of smoothing, the pre-RC binding sites can generally be localized at a resolution that is comparable to the resolution of the microarray. By providing more defined sites, these studies have been useful in computational studies to predict sites of replication initiation in yeast cells (see below).

The major disadvantage of the genome-wide location analysis approach is that the sites of 
pre-RC assembly only mark potential origins of replication. These studies do not actually look at replication products and therefore provide no information concerning which pre- $\mathrm{RC}$ complexes initiate in a given cell cycle. Indeed, direct tests of the pro-ARSs identified showed that not all are used as origins (even in a plasmid context), suggesting that there is a significant number of false positives in these data. For example, of the 429 identified pro-ARSs, at least 10 have been directly shown to be false positives. In addition, these studies cannot describe the temporal pattern of replication or the efficiency of any given origin.

It is important to note that none of these methods has achieved the ultimate goal of defining the DNA sequences that are required for each origin's function (i.e. the replicator sequences). As in most cases, it is likely that the best definition of origins will come from combining the results obtained from multiple approaches.

\section{Comparison of yeast origin mapping techniques}

The three studies mapping yeast origins of replication, despite using different technologies and $S$. cerevisiae strains, have a high degree of overlap. The density transfer and copy number approaches identified 332 and 247 origins, respectively. The identification of pre-RC binding sites along the yeast genome revealed 429 potential origins of replication. We have assembled all three data sets and queried them for overlap between predicted origins. To account for the different resolutions of the studies, we have conservatively called an identified locus overlapping if it was within $5 \mathrm{~kb}$ of an identified locus in the other data sets. The Venn diagram in Figure 1A summarizes the overlap observed between the three data sets. Although both pre-RC localization and density transfer data sets have a large number of unique origins not identified in the other experiments, only 37 of the origins identified by the copy number method, are not present in either of the two other data sets. This suggests that the copy number data set is the most conservative of the three and probably has the lowest number of false positives. Indeed, as described above, the nature of the pre-RC mapping will inherently have a high degree of false positives.
Using the intergenic co-ordinates of the proARSs identified by genome-wide location analysis, we have assembled a table (Supplementary Table $\mathrm{S} 1 *$ ) that lists the location of the origins confirmed in all three data sets and their respective time of replication from either of the replication profile studies. These identified origins, termed 'array-based origins' (ABOris), contain both early- and (potential) late-activating origins (Figure 1B).

The site of replication initiation for these origins can only be resolved down to the resolution of the array used for genome-wide location analysis, in this case approximately 500 bases. Although these techniques can be used to locate origins of replication and characterize their temporal pattern of activation, it is important to emphasize that they clearly do not have the resolution to identify an essential replicator sequence. Breier et al. have developed a computer algorithm, 'oriscan', to identify ACS elements that direct the initiation of replication (Breier et al. 2004). Because of the degenerate nature of the ACS there are potentially 12000 sites in the yeast genome that match the ARS consensus sequence (approximately 1 ACS/ $1000 \mathrm{bp}$ ). Using a training set of 26 experimentally characterised origins, the oriscan algorithm identified a unique sequence signature present in these defined origins. Applying this algorithm to the entire genome, the authors identified 350 candidate ACS sequences. Although the authors found that $37 \%$ of the pro-ARSs are identified by oriscan, we found that $45 \%$ of the ABOs were identified by oriscan (Supplementary Table S1).

\section{Insights into replication of yeast chromosomes}

What have we learned from the large-scale systematic identification of replication origins in $S$. cerevisiae? These studies indicate that the temporal pattern of replication is clearly influenced by the architecture of the chromosome. As had been observed for a limited number of chromosomes (Reynolds et al. 1989, Newlon et al. 1993, Friedman et al. 1996, Friedman et al. 1997), these studies also confirmed that, in contrast to many other eukaryotic organisms, the sequences

*Available online at http://www.kluweronline.com/issn/0967-3849/ 
surrounding each centromere are replicated early in $\mathrm{S}$ phase. In contrast, the subtelomeric regions consistently replicate later than the rest of the genome. It is important to note that, while the telomeric sequences replicate late, they are not necessarily always the latest replicating sequences. One novel observation from the heavy:light replication timing profiles was that the replication of the ends of each chromosome were coordinated. The subtelomeric sequences of each chromosome replicated at almost precisely the same time, yet, as a whole, different pairs of chromosome telomeres were not co-ordinated. Recent studies in human cells (Zou et al. 2004), however, have observed that the subtelomeric regions for each chromosome replicate independently of each other and are not co-ordinated. Careful examination of the yeast replication timing data suggests that the similar time of replication observed for each chromosome's telomeres could be a result of circularizing the chromosomal co-ordinates to generate a continuous data set for the smoothing algorithms. The biological significance of this observation therefore remains to be determined.

Potential origins of replication are marked by ORC binding throughout the cell cycle and ORC functions as a molecular machine to assemble the rest of the pre-RC at the end G1 (reviewed in Mendez \& Stillman 2003). The assembly of the pre-RC and the temporal activation of origins are distinct steps in replication of a chromosome. The comprehensive survey of the genome for pro-ARSs revealed that the pre- $\mathrm{RC}$ is primarily formed in intergenic sequences, consistent with earlier studies of origins. The density of pro-ARS sites was increased at the telomeres with up to 5 adjacent pro-ARS sites within $20 \mathrm{~kb}$ of the end of the chromosome. Pro-ARS sequences coincided at a higher frequency than expected with repetitive elements such as transposable elements, long terminal repeats and $t R N A s$.

Does transcription help to define yeast origins of replication? All yeast genomic replication studies have reported that no direct correlations were observed between steady-state transcription levels and either the establishment or the activation of origins. A recent applied mathematics paper (Alter $\&$ Golub 2004), using the pro-ARS data set identified by genome-wide location analysis, coupled with analysis of the transcriptome through the cell cycle (Spellman et al. 1998), reported a correlation between sites of pre-RC formation and the shutdown of adjacent genes during G1. The significance (and predictive value) of this correlation remains unclear; does the assembly of the pre-RC at G1 retard transcription or does active transcription retard the assembly of the pre-RC? Alternatively, the perceived changes may be due to system-wide oscillations in the underlying redox state of the cell (Klevecz et al. 2004). At high cell densities the yeast cell population begins regular oscillations in respiratory potential. Three discrete maximums of transcription are observed for the majority of transcripts spaced at clear temporal intervals during the respiratory oscillations: one during the oxidative phase of the oscillation and two distinct pulses delineating the beginning and end of the reduction phase. Interestingly, entry into $\mathrm{S}$ phase is precisely gated to the reduction phase of the cycle and, thus, co-ordinated with the expression of a large number of genes.

Although the influence of active transcription (as measured by steady-state mRNA levels) on the process of replication is unclear, the organization of genes and the direction of transcription may influence the sites that are selected to act as origins of replication. The pro-ARSs identified by genomewide location analysis were almost exclusively found in intergenic sequences. We have re-analysed these intergenic sequences and have discovered a striking correlation between the direction of transcription of neighbouring ORFs and pre-RC assembly. Significantly fewer pro-ARSs are found in intergenic sequences flanked by diverging transcripts than would be expected based on the distribution of the entire population (Table 1). Specifically, only $10 \%$ of identified pro-ARSs are located between diverging transcripts. In contrast, in $S$. pombe, it has been shown that origins are preferentially localized between divergent transcripts (Segurado et al. 2003). It remains to be determined if these differences represent fundamentally distinct ways by which ORC is recruited to the DNA in $S$. cerevisiae and $S$. pombe.

There are several possible reasons to account for finding fewer pre-RCs assembled at divergent transcripts. First, the size of the intergenic region may spatially dictate whether a pre-RC can assemble. For example, intergenic regions which assemble pre-RC are significantly larger than that of the 
A.

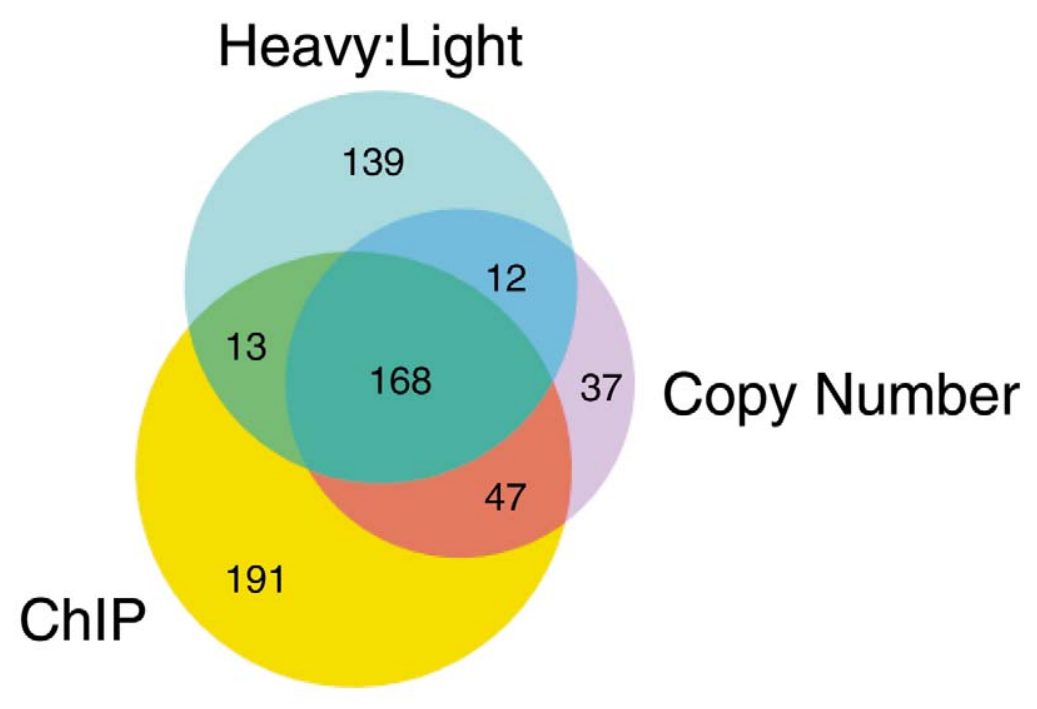

B.

Array Based Origins (ABOs) on Chr IV

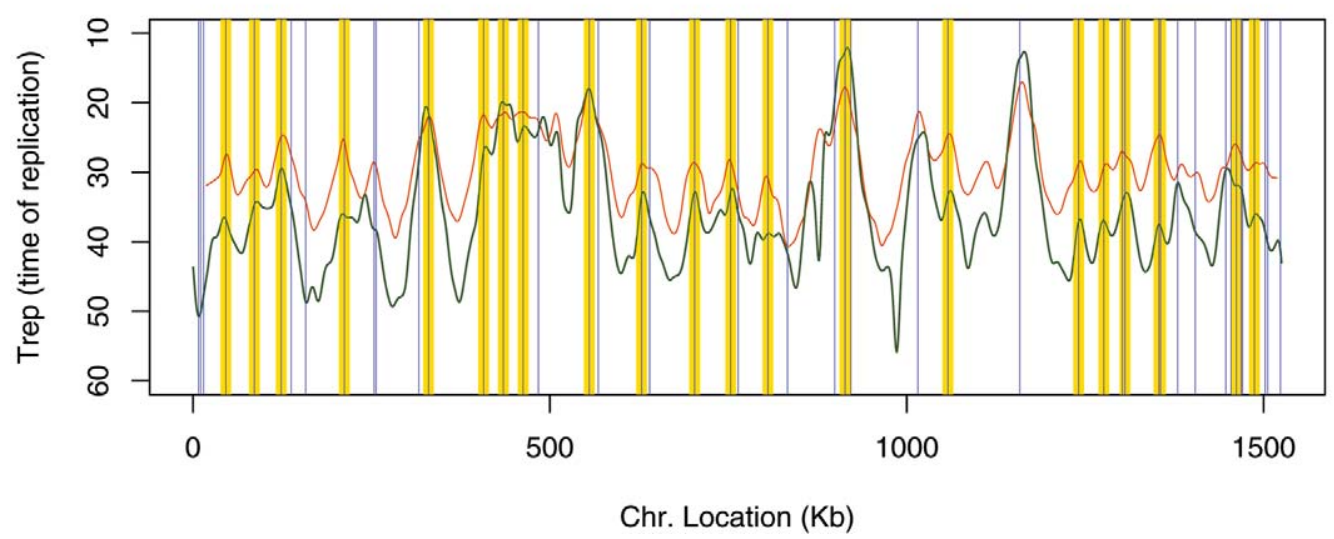

Figure 1. Comparison of S. cerevisiae origin mapping techniques. (A) Venn diagram depicting the overlap in origin prediction between the three methods to identify yeast origins of replication: genome-wide location analysis (ChIP), heavy: light, and copy number. The data sets were considered overlapping if the predictions were within $5 \mathrm{~kb}$ of each other. Because many of the origins identified by the timing methods may potentially overlap with multiple pro-ARSs, we only scored an overlap between distinct origins as predicted by the timing data. Hence the amount of overlap in the genome-wide location analysis is under-estimated. The online data from the copy number experiments did not contain the origin predictions of the 247 origins. Using their described algorithm and data, we identified 264 origins, not 247. (B) Map of array-based origins (ABOs) for chromosome IV. Replication timing profiles from the heavy:light (green line) and copy number (red line) experiments were plotted as Trep (time of replication) versus chromosomal location. pro-ARSs identified by the genome-wide location analysis experiments are indicated by vertical blue lines. The locations of the $\mathrm{ABOs}$ are indicated by vertical yellow lines. Two likely $\mathrm{ABOs}$ (at $1 \mathrm{Mb}$ and $1.15 \mathrm{Mb}$ ) are missed due to the conservative nature of the 5-kb cut-off. A full list of ABOs can be found in Supplementary Table S1 and additional maps of ABOs for all the yeast chromosomes are available at http://bell-lab-server.mit.edu/ABOrimaps. 
population (Wyrick et al. 2001). Thus, if the intergenic regions between divergent transcripts were smaller than found between convergent transcripts, it might explain the observed bias. However, just the opposite is true. The mean size of the intergenic regions between divergent transcripts is significantly larger than that of the population (Table 1). Second, the assembly of transcription factors near the promoters of divergent genes may compete with pre-RC components for DNA occupancy. Nucleosomes positioned by these factors could interfere with pre-RC assembly (Lipford \& Bell 2001). Finally, the direction of transcription of neighbouring sequence may change the local topology of the intervening intergenic DNA, possibly resulting in increased negative supercoils between divergent transcripts. Interestingly, in Drosophila, it has been shown that ORC binds preferentially to negatively supercoiled DNA (Remus et al. 2004) that would be expected to form between divergent genes.

\section{Characterization of metazoan replication}

The size and complexity of metazoan genomes has hindered the identification and characterization of their origins of replication. Genes, telomeres, centromeres and origins of replication are all larger and more complex than their single-cell eukaryotic counterparts. The more extensive presence of repetitive sequences makes analysis of replication origins more difficult. The plasmid-based approaches that were so successful in identifying $S$. cerevisiae origins of replication have proven unsuccessful for metazoan organisms. This lack of success is in part due to the large size of centromeres preventing their inclusion on plasmids to allow stable segregation. The most successful of the attempts to identify origins using plasmid-based studies found that the size, rather than sequence, of the metazoan DNA present in the plasmid most closely correlated with plasmid stability (Heinzel et al. 1991).

Only a handful of metazoan origins have been studied in detail. In general, these origins have been identified using sensitive methods that detect replication intermediates generated during replication initiation (reviewed in Gerbi 2004). A subset of these origins can be moved to ectopic sites in the genome and retain the ability to direct replication initiation, indicating that they are true replicator sequences (reviewed in Biamonti et al. 2003, Aladjem \& Fanning 2004). These origins tend to span relatively small regions of DNA $(6 \mathrm{~kb})$ and, in at least one case, specific sequence elements that are important for the function of the origin in an ectopic location have been identified (Altman \& Fanning 2004). Other origins span much larger regions (greater than $10 \mathrm{~kb}$ ) and include multiple sites of initiation (reviewed in Bogan et al. 2000). In several instances, sequences well away from the actual start site of replication influence either the efficiency or timing of metazoan origins intiation. Finally, despite the conservation of pre- $\mathrm{RC}$ factors in all eukaryotes, no essential origin consensus sequence analogous to the $S$. cerevisiae ACS has been identified for any metazoan genome.

The small number of metazoan origins that have been identified and studied has not revealed how they are selected or activated. It remains unclear whether specific sequences, adjacent transcription or other chromosomal features (e.g. local chromatin structure) are the primary determinants of origin selection. Although early studies of metazoan DNA replication showed that highly transcribed euchromatic regions replicated early and poorly transcribed heterochromatic regions replicated late (Goldman et al. 1984), the connection between transcriptional status and origin activation also remains poorly understood.

Five recent studies that are reviewed below have applied genomic approaches, similar to those described for $S$. cerevisiae, to shed light on the replication of metazoan genomes. Comparisons of replication timing profiles and transcription profiles across genomes or chromosomes have revealed important information about the level at which transcription and replication is coupled. A subset of these studies have also been able to identify novel origins of replication in both human and Drosophila cells.

\section{Metazoan replication timing}

The first metazoan replication profile was generated using Drosophila Kc tissue culture cells (Schübeler et al. 2002). Because, until recently (see below), Kc cells were not thought to be amenable to cell synchronisation, the authors labelled actively replicating DNA sequences in an 
Table 1. ProARS sequences are enriched at convergent transcripts rather than divergent transcripts. For each set, total (all intergenic sequences), pro-ARS (intergenic pro-ARS sequences) and ABO (array-based origins), the average size of the intergenic region and the observed number of flanking transcripts are reported. Three classes of transcripts are noted, transcripts running in tandem $(\rightarrow \rightarrow$, either direction), divergent transcripts $(\leftarrow \rightarrow)$ or convergent transcripts $(\rightarrow \leftarrow)$. The expected numbers are based on the total population. The $\chi^{2}$ and $p$ values are indicated.

\begin{tabular}{|c|c|c|c|c|c|c|}
\hline Set & Transcripts & Size & Observed & Expected & $\chi^{2}$ & $p$ \\
\hline \multirow{3}{*}{ Total } & $\rightarrow \rightarrow$ & 509 & 3557 & & & \\
\hline & $\leftarrow \rightarrow$ & 559 & 1350 & & & \\
\hline & $\rightarrow \leftarrow$ & 314 & 1384 & & & \\
\hline \multirow{3}{*}{ pro-ARS } & $\rightarrow \rightarrow$ & 721 & 195 & 196 & & \\
\hline & $\leftarrow \rightarrow$ & 889 & 35 & 72 & 39.31 & $2.91 \times 10^{-9}$ \\
\hline & $\rightarrow \leftarrow$ & 459 & 113 & 75 & & \\
\hline \multirow{3}{*}{$\mathrm{ABO}$} & $\rightarrow \rightarrow$ & 756 & 81 & 84 & & \\
\hline & $\leftarrow \rightarrow$ & 793 & 17 & 30 & 17.01 & $2.02 \times 10^{-5}$ \\
\hline & $\rightarrow \leftarrow$ & 487 & 49 & 32 & & \\
\hline
\end{tabular}

asynchronous culture using a pulse of BrdU. Flow cytometry was used to isolate the BrdUlabelled cells at either the beginning or end of $\mathrm{S}$ phase based on their FACS profile. DNA was isolated from the early and late S-phase cell populations, and replicated DNA was specifically enriched by immunoprecipitation using BrdUspecific antibodies. The purified early- and latereplicating DNA populations were differentially labelled with fluorescent nucleotides and hybridized to cDNA based microarrays representing $40 \%$ of the predicted Drosophila genes. The ratio of early- and late-replicating DNA for each cDNA feature was used to generate a replication timing profile for this data. Because the majority of cDNAs found on the microarray are located in the euchromatin, this study was limited to this portion of the genome for the most part. However, analysis of several transposable elements that were exclusive to the heterochromatin revealed that they replicated later than $98 \%$ of analysed euchromatic sequences.

A higher resolution replication timing profile of only the left arm of Drosophila chromosome 2 took advantage of a custom microarray that covered almost $90 \%$ of the euchromatic sequence of the chromosome arm with nearly overlapping 1.5-kb PCR products (MacAlpine et al. 2004). For these studies, Kc cells were synchronized using a two-step protocol. First, the cells were arrested in G2 by the addition of the molting hormone Ecdysone. Second, the cells were released from the ecydysone block into arresting concentrations of
$\mathrm{HU}$, resulting in a synchronized population of cells arrested at the G1/S transition. The cells were released from $\mathrm{HU}$ and allowed to proceed synchronously through $\mathrm{S}$ phase. Early-replicating sequences were labelled using a pulse of BrdU at the onset of S-phase and similarly late-replicating sequences were labelled with a pulse of BrdU near the end of S phase. The early and late DNA samples were differentially labelled and applied to the array, and the ratio of early to late replicating sequences for each DNA feature was used to generate a replication timing profile. The replication timing curve for chromosome $2 \mathrm{~L}$ generated by this method showed significant similarity with that of the previous Drosophila study that used a cDNAbased microarray (Schübeler et al. 2002).

The first replication profile of the entire human genome was generated from lymphoblastoid cells (Woodfine et al. 2004). In this study, fluorescenceactivated cell sorting was used to separate asynchronous cell populations into either a G1 or a single actively replicating S-phase population. DNA from these cell populations were differentially labelled with fluorescent nucleotides and hybridized to genomic microarrays. Two types of genomic microarrays were used in these experiments: one containing $150 \mathrm{~kb}$ probes spaced at $1-\mathrm{Mb}$ intervals across the entire genome, and a second higher-resolution array (various sized probes that were located approximately every $78 \mathrm{~kb}$ ) that covered the q arm of chromosome 22. Analogous to the yeast copy number studies using pooled S-phase samples (Yabuki et al. 2002), the DNA near origins that 
initiate replication early in S-phase was overrepresented in the S-phase pool and, conversely, the DNA that replicated later in $\mathrm{S}$ phase was under-represented. In contrast, the copy number of G1 DNA should be uniform across the genome. Thus, the relative ratio of $\mathrm{S}$ to G1 DNA for a given locus was considered indicative of its relative time of replication and was used to generate a replication timing profile.

Two more recent studies have also examined replication timing in human cells. One study examined replication timing in Hela cells using an ultrahigh density Affymetrix array that 'tiled' chromosomes 21 and 22 with small oligonucleotides (approximately 1 oligonucleotide per $35 \mathrm{bp}$; A. Dutta, personal communication). Cell-cyclesynchronized HeLa cells were pulsed with BrdU during six different time intervals during $\mathrm{S}$ phase to label replicating DNA. The resulting BrdUlabelled DNA was enriched by $\mathrm{CsCl}$ centrifugation, labelled with fluorescent nucleotides and hybridized to multiple microarrays. The resulting data were then used to generate a replication timing profile. A second study compared replication timing in two human cells: lung fibroblasts and a lymphoblastoid cell line (White et al. 2004). This study used a custom array that 'tiled' chromsome 22 with nearly overlapping amplified PCR fragments (average size of $700 \mathrm{bp}$ ). The replication timing for these cells was established using similar methods to those of the Drosophila cDNA study (Schübeler et al. 2002).

\section{Replication timing and transcription}

Each of the metazoan replication timing profiles described above was compared to at least one type of transcription profile to explore any connection between transcription and replication timing. In the case of the Drosophila cDNA study, a comparison between mRNA message levels and replication timing for each cDNA showed no clear correlation (Schübeler et al. 2002). A more detailed statistical analysis did reveal that the probability of a given gene being expressed (that is, whether the gene exceeded a minimal detectable threshold of expression) was a function of replication timing. Specifically, there was a decreased probability of finding an expressed gene in the latest replicating regions of the genome and an increased probability of finding an expressed gene in the earliest replicating regions. It is important to note that not all expressed genes were replicated early and that not all nonexpressed genes replicated late. Put simply, whether or not a given gene was expressed had little predictive value for replication timing; instead, only the regions of the genome that were replicated very early or very late had predictive value for gene expression.

A similar correlation between gene expression and replication timing was observed in the human genomic studies (Woodfine et al. 2004). The expression pattern of 1300 human genes (which overlapped with the large clones of the $1 \mathrm{Mb}$ array) was examined. As with the Drosophila cDNA studies, no correlation was observed between replication timing and levels of transcription but the probability of a gene being expressed was greater for the earlier replicating clones. These studies also noted a positive correlation between replication timing and gene density. Both gene-dense and GCrich regions of the human genome were found to replicate before regions of low gene density, suggesting a link between replication and gene distribution along the chromosome. Similar observations were also made for the other human studies (White et al. 2004, Jeon et al. 2005). Taken together with the results from the Drosophila cDNA study (Schübeler et al. 2002), it appears that the coupling of replication timing and transcription is conserved in higher eukaryotes.

To look more carefully at the connection between replication timing and transcription, the high-resolution Drosophila genomic array was used to identify sites of active transcription along the chromosome arm (MacAlpine et al. 2004). Sites of active transcription were identified along the chromosome using genome-wide location analysis of RNA polymerase II, dimethylated histone $\mathrm{H} 3 \mathrm{~K} 4$, and mRNA expression analysis. RNA pol II and dimethylated histone $\mathrm{H} 3 \mathrm{~K} 4$ localization proved to be accurate predictors of mRNA expression (Schübeler et al. 2004, MacAlpine et al. 2004). As with the studies described above, there was little correlation between replication timing and the level of expression of immediately adjacent genes. Interestingly, if the density of RNA Pol II association was determined over larger 
windows of sequence, a clear correlation with replication timing emerged. In fact, the latest decile of replicating sequences was an order of magnitude less likely to contain sites of active transcription than the earliest replicating sequences. This level of correlation between RNA Pol II density and replication timing reached a clear peak when the window used to calculate RNA Pol II density reached $180 \mathrm{~kb}$ (a similar pattern was observed for other measures of gene expression including chromatin modification and mRNA expression). This observation suggests that replication timing is linked most strongly to transcription status integrated over large regions rather than the individual loci, predicting that changes in the expression of an individual gene are unlikely to be associated with a change in replication timing (see below).

Analysis of the high-density Drosophila microarray studies also suggested that the boundaries of replication timing domains are likely to be connected to boundaries of transcription domains. Comparison of a map of the local density of RNA Pol II along the chromosome arm with the replication timing profile revealed that the transition between early- and late-replicating domains frequently correlated with dramatic shifts in the local RNA Pol II density (Figure 2). Interestingly, the height of the associated peaks in the replication timing profile correlated less well with the absolute levels of associated RNA Pol II density. That is, a transition from low to medium to low RNA Pol II density can just as easily be associated with a peak of early replication as a transition from medium to high to medium RNA Pol II density. These data suggest that it is not the absolute level of transcription, but rather localized regions of elevated transcription in the chromosome that define replication timing domains. These transcription/replication timing domains could be defined by higher-order chromosome structure, such as boundary elements, transcriptional insulators or elements of higher-order chromatin structure (e.g. chromosome loops).

\section{Identification of metazoan origins of replication}

The human study using an ultrahigh resolution array identified at least 400 genomic sites at which replication appears to initiate at discrete
S-phase intervals (A. Dutta, personal communication). These sites were defined as regions in which the total hybridization signal for all probes in a $10-\mathrm{kb}$ window was significantly enriched. Those sites identified in the earliest S-phase interval are most likely to contain origins of replication. It is difficult to determine whether the discrete sites of replication identified at the later S-phase intervals are the result of de-novo origin activation or the result of fork progression. One of the most interesting observations from this data set was that $60 \%$ of the genome appeared to incorporate BrdU equally efficiently in all $\mathrm{S}$ phase intervals examined, suggesting that the majority of human DNA replication may result from inefficient asynchronous origins located throughout the genome.

Because studies in $S$. cerevisiae suggested that origin identification by multiple techniques increases the confidence of the prediction, three methods were used to identify replication origins using the high-resolution Drosophila chromosome 2L microarray: replication timing, identification of $\mathrm{HU}$ resistant early origins, and ORC localization. Replication origins were first localized to broad regions $(50-100 \mathrm{~kb})$ using replication timing data. The lack of temporal resolution of these studies was probably a function of the less than complete synchrony of the cell populations. To define a subset of the origins at higher resolution, cells were allowed to enter $\mathrm{S}$ phase synchronously in the presence of $\mathrm{HU}$ and BrdU. As described previously for S. cerevisiae (Yabuki et al. 2002), this treatment should allow BrdU incorporation only at sequences immediately adjacent to early origins of replication. The BrdU-labelled DNA was purified and hybridized to the array allowing the identification of 62 early activating origins. No early HU-resistant origins of replication were found in the late-replicating domains, consistent with the intra S-phase checkpoint functioning in Drosophila cells.

Origin localization was further refined by identifying candidate ORC binding sites using genomewide location analysis. 491 ORC binding sites were identified, many of which localized to earliest sites of replication, most often at the apex of BrdU incorporation. In contrast to $S$. cerevisiae, where clustering of ORC is seen only at subtelomeric sequences (Wyrick et al. 2001), 20\% of the 


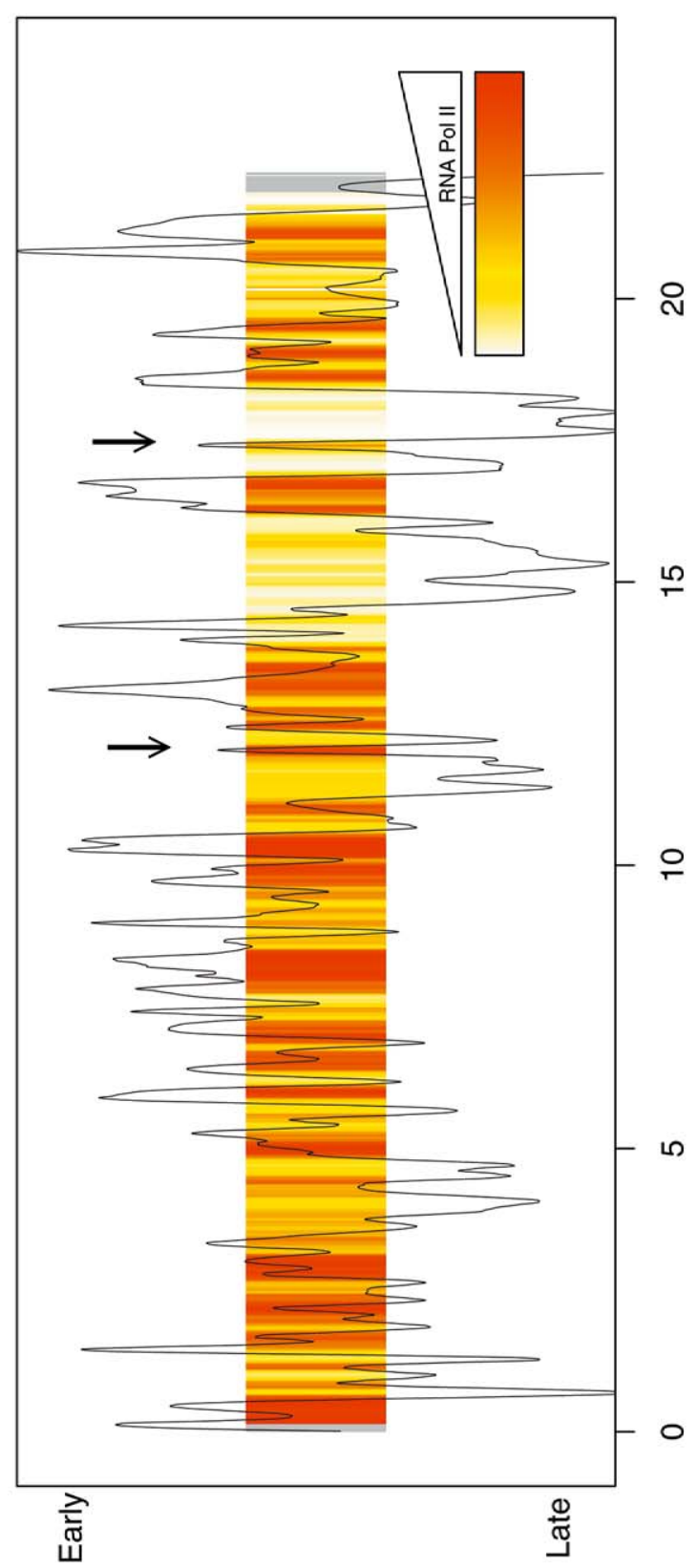

6u!m!!

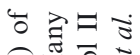

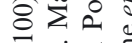

II

$\therefore$ 落

훙 $\frac{\pi}{2}$

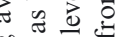

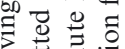

을 율.

\& $\approx$

0 政

은 矛趇

다이

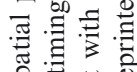

की

을

造

音昰

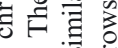

छ䑻

舫

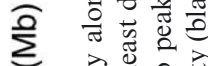

대응

专 专完

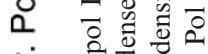

立艺范范

告

\% 尊

퀀ㅇㅇ

$\exists . \Xi$

范荡

च స 등

它合

.

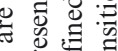

놀 छ

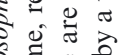

हैं है

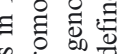

킁 굴

苛 艺言

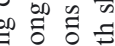

은 $\frac{0}{60}$

苟

을 苞

음

블

i $\overline{0}$

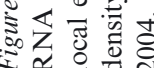


Drosophila ORC binding sites were immediately adjacent to other ORC sites. The median distance between non-adjacent ORC sites was approximately $25 \mathrm{~kb}$, consistent with published estimates of the Drosophila replicon (Blumenthal et al. 1974). Regions of clustered ORC binding could be associated with replication initiation zones, similar to those described in the analysis of metazoan replication origins in the past (reviewed in Bogan et al. 2000 ) but generally not observed in $S$. cerevisiae.

The identification of 491 ORC-binding sites provided an opportunity to investigate the molecular determinants of ORC-binding metazoa. As yet, there is no known DNA-binding site for metazoan ORC (equivalent to the ACS in $S$. cerevisiae). Analysis of the 491 ORC-binding sites did not identify such a consensus sequence. It is interesting to note that application of the same algorithms to the genome-wide location analysis of $S$. cerevisiae ORC were unable to identify the ACS, suggesting that the resolution of these studies is not yet at a level to identify binding sites. These findings are distinct from the oriscan studies which started with the knowledge of the sequence of the ACS and used the genome-wide location studies to select particular copies of the ACS that were most likely to be associated with origins. The Drosophila ORC-associated sequences were significantly more AT-rich than the genome as a whole, consistent with the preferential association of Drosophila ORC with AT-rich sequences in vitro (Chesnokov et al. 1999).

Interestingly, a large fraction of ORC sites colocalized with a subset of the RNA Pol II binding sites. The RNA Pol II sites associated with ORC were enriched at the $5^{\prime}$ end of genes consistent with these sites being near transcription promoters. Further experiments found no evidence for a direct interaction between RNA Pol II and ORC. Instead, it seems more likely that ORC is recruited to the DNA through the action of various transcription factors (Bosco et al. 2001, Danis et al. 2004) and RNA Pol II serves only as a marker for sites of transcription factor binding. Such transcription factor-dependent recruitment of ORC could be due to direct interaction with transcription factors or to indirect effects resulting from the establishment of permissive chromatin structures by the action of the transcription factors.

\section{Developmental and tissue-specific control of replication}

The process of replication in higher metazoans is dynamic and able to respond to various tissuespecific and developmental programs. A classic example is the increased origin density observed during early Xenopus embryogenesis when $\mathrm{S}$ phase typically only lasts for a few minutes (Hyrien et al. 1995). Another example is the tissue specific amplification of four discrete loci in Drosophila follicle cells during oogenesis (reviewed in Edgar \& Orr-Weaver 2001). Two of these loci have been localized to the third and X chromosomes and each encodes a family of proteins related to chorion egg shell production. Studies of the chorion locus on chromosome $3 \mathrm{~L}$ identified a well-defined origin of replication that has served as a paradigm for metazoan origin function (reviewed in Calvi \& Spradling 1999). Despite extensive characterization of the amplification of the two chorion loci, the two remaining amplification loci were unidentified. Recent experiments used a Drosophila cDNA-based microarray to survey DNA copy number across the genome, from follicle cells that had undergone amplification, to identify the remaining loci (Claycomb et al. 2004). DNA from the amplified tissue was fluorescently labelled and hybridized against control non-amplified DNA on a cDNA microarray. The chorion loci were readily detectable, as were the two additional loci. The location of the new amplicons was confirmed by fluorescent in-situ hybridization (FISH). Interestingly, one of these loci was shown to be proximal to a gene required for viable eggs, suggesting that all of the follicle cell amplicons are involved in egg shell formation.

Is there developmental control of the temporal pattern of replication? Previous studies of individual loci have indicated that changes in gene expression can be associated with changes in the local pattern of replication (reviewed in Gilbert 2001). To look at this possibility in a more comprehensive manner, a high-resolution replication timing profile for human chromosome 22 was determined for two different human cell types: one derived from lung fibroblasts and a second from lymphoblastoid cells (White et al. 2004). Interestingly, despite the presence of many genes that were 
differentially expressed between the two cell types, more than $99 \%$ of the sequences analysed exhibited indistinguishable replication timing profiles. One of the few regions that showed both a significant change in replication timing and gene expression between the two cell types included the Ig $\lambda$-like locus. This locus is more highly expressed and replicates earlier in the lymphoid cells. Interestingly, several of the previously characterized regions of the human genome that show dramatic changes in replication timing in response to changes in gene expression include genes associated with the immune system (Mostoslavsky et al. 2001). One noteworthy feature of the regions that change timing when their genes are activated is their complexity: the genes and their regulatory sequences frequently span $>100 \mathrm{~kb}$. Based on the finding in Drosophila Kc cells that replication timing is linked most closely to transcriptional activity integrated over large regions rather than individual loci, activation of such complex loci may be more likely to be coupled to changes in replication timing.

\section{Summary}

The ability to assess the dynamics of DNA replication at whole-genome and chromosome level has provided new insights into the global regulation of DNA replication. These studies clearly illustrate both the reproducibility and the complexity of the replication process in eukaryotic cells. Different approaches, such as replication timing and genome-wide location analysis, have been used to identify origins of replication and characterize their temporal pattern of activation in a variety of eukaryotic cell types. These studies have also made clear that the organization of the chromosome influences origin activation. For example in yeast, sequences surrounding the centromeres are replicated early and a recurring theme in studies from higher eukaryotes is that gene-dense actively transcribed regions are replicated prior to gene-sparse regions of the chromosome.

Although the approximate location of replication origins can be determined by the current methodologies, the resolution is not yet sufficient to identify key DNA elements that are required for origin function. Even in S. cerevisiae where such sites were previously identified by analysis of a small subset of origins, the microarray studies have not yet reached the resolution to independently identify such sequences as the ORC-binding site (the ACS). Increased array resolution and the use of multiple technologies have the promise of refining the resolution of these studies to a point that should allow the identification of such sequences if they are present and non-redundant. In metazoan organisms, there is currently only limited evidence for the presence of such elements. Nevertheless, the reproducible patterns of replication observed in Drosophila and human tissue culture cells strongly suggests that there are mechanisms that restrict the initiation of replication to particular regions of the genome and particular times in $\mathrm{S}$ phase. In the long term, the findings of microarray studies of DNA replication will have to be combined with genetic and biochemical studies to fully characterize the determinants of this pattern. However, the availability of large numbers of DNA sites that act as origins of replication will be essential for these studies to determine the mechanisms that direct pre-RC formation and origin activation in metazoan organisms.

The future of genomic microarray studies of replication will be to generate novel mechanistic insights into regulation of genomic replication. The experiments described in this review have generally been of a descriptive nature, establishing correlations between replication timing and chromosomal features as well as establishing a catalogue of identified origins for future study. Although these studies provide important new tools for the genetic analysis of origins and restrict the type of mechanisms that could account for the connection between replication and transcription, they have yet to provide true mechanistic insights. As these approaches become part of the array of tools to assess DNA replication, they will contribute to our mechanistic understanding of replication. For example, a recent study using a high-density oligonucleotide array of chromosome VI in S. cerevisiae provided insights into the function of the S-phase checkpoint proteins, Tof1 and Mrc1 (Katou et al. 2003). Although both proteins were known to be required for the intra-S-phase checkpoint, their mechanism of function was poorly understood. Using genome-wide location analysis, the authors of this study found that both proteins 
are components of the DNA replication fork and remain associated with the fork after it stalls, presumably to signal a defect in replication fork progression.

Combining microarray-based assays of replication with genetic analysis will undoubtedly provide important insights into the mechanisms that control the co-ordination and regulation of eukaryotic replication. Although all eukaryotic organisms maintain a clear pattern of replication timing across their genome, the mechanisms responsible for establishing this conserved and characteristic aspect of each cell's replication remain obscure. The ability of microarray-based assays to easily monitor changes that occur when candidate replication timing regulatory genes are inhibited (either by mutation or by RNAi) will be a powerful new tool for understanding the temporal regulation of replication. The finding that this type of regulation reaches beyond the function of individual genes and may be particularly responsive to changes in gene expression that extend over large domains suggests that such studies are likely to provide insights beyond the activation of origins, potentially extending to the proteins that establish the global architecture of chromosomes and their influence on genomic function.

\section{Acknowledgements}

We thank Hannah Blitzblau, Milan de Vries, Cary Lai, and Dirk Schübeler for comments and discussion. We are especially thankful to M. K. Raghuraman for insightful discussions regarding the time of yeast telomere replication and A. Dutta for sharing unpublished data. The work in S.P.B.'s laboratory is supported by the Howard Hughes Medical Institute and the National Institutes of Health (GM52339). D.M.M. is a fellow of the Damon Runyon-Walter Winchell Cancer Fund.

\section{References}

Aladjem M, Fanning E (2004) The replicon revisited: an old model learns new tricks in metazoan chromosomes. $E M B O$ Rep 5: 686-691.

Alter O, Golub G (2004) Integrative analysis of genome-scale data by using pseudoinverse projection predicts novel correlation between DNA replication and RNA transcription. Proc Natl Acad Sci USA 101: 16577-16582.

Altman A, Fanning E (2004) Defined sequence modules and an architectural element cooperate to promote initiation at an ectopic mammalian chromosomal replication origin. $\mathrm{Mol}$ Cell Biol 24: 4138-4150.

Anglana M, Apiou F, Bensimon A, Debatisse M (2003) Dynamics of DNA replication in mammalian somatic cells: nucleotide pool modulates origin choice and interorigin spacing. Cell 114: 385-394.

Aparicio J, Viggiani C, Gibson D, Aparicio O (2004) The Rpd3-Sin3 histone deacetylase regulates replication timing and enables intra-S origin control in Saccharomyces cerevisiae. Mol Cell Biol 24: 4769-4780.

Bell S (1995) Eukaryotic replicators and associated protein complexes. Curr Opin Genet Dev 5: 162-167.

Bell S (2002) The origin recognition complex: from simple origins to complex functions. Genes Dev 16: 659-672.

Bell S, Marahrens Y, Rao H, Stillman B (1993) The replicon model and eukaryotic chromosomes. Cold Spring Harb Symp Quant Biol 58: 435-442.

Biamonti G, Paixao S, Montecucco A, Peverali F, Riva S, Falaschi A (2003) Is DNA sequence sufficient to specify DNA replication origins in metazoan cells? Chromosome Res 11: 403-412.

Blumenthal A, Kriegstein H, Hogness D (1974) The units of DNA replication in Drosophila melanogaster chromosomes. Cold Spring Harb Symp Quant Biol 38: 205-223.

Bogan J, Natale D, Depamphilis M (2000) Initiation of eukaryotic DNA replication: conservative or liberal? J Cell Physiol 184: 139-150.

Bosco G, Du W, Orr-Weaver T (2001) DNA replication control through interaction of E2F-RB and the origin recognition complex. Nat Cell Biol 3: 289-295.

Bowers J, Randell J, Chen S, Bell SP (2004) ATP hydrolysis by ORC catalyzes reiterative Mcm2-7 assembly at a defined origin of replication. Mol Cell 16: 967-978.

Breier A, Chatterji S, Cozzarelli N (2004) Prediction of Saccharomyces cerevisiae replication origins. Genome Biol 5: R22.

Brewer B, Fangman W (1987) The localization of replication origins on ARS plasmids in S. cerevisiae. Cell 51: 463-471.

Brewer B, Fangman W (1991) Mapping replication origins in yeast chromosomes. Bioessays 13: 317-322.

Calvi B, Spradling A (1999) Chorion gene amplification in Drosophila: A model for metazoan origins of DNA replication and S-phase control. Methods 18: 407-417.

Chesnokov I, Gossen M, Remus D, Botchan M (1999) Assembly of functionally active Drosophila origin recognition complex from recombinant proteins. Genes Dev 13: 1289-1296.

Claycomb J, Benasutti M, Bosco G, Fenger D, Orr-Weaver T (2004) Gene amplification as a developmental strategy: isolation of two developmental amplicons in Drosophila. Dev Cell 6: $145-155$.

Danis E, Brodolin K, Menut S, Maiorano D, Girard-Reydet C, Mechali M (2004) Specification of a DNA replication origin by a transcription complex. Nat Cell Biol 6: 721-730.

Donaldson A, Raghuraman M, Friedman K, Cross F, Brewer B, Fangman W (1998) CLB5-dependent activation of late replication origins in S. cerevisiae. Mol Cell 2: 173-182. 
Edgar B, Orr-Weaver T (2001) Endoreplication cell cycles: more for less. Cell 105: 297-306.

Friedman K, Diller J, Ferguson B, Nyland S, Brewer B, Fangman W (1996) Multiple determinants controlling activation of yeast replication origins late in $\mathrm{S}$ phase. Genes Dev 10: 1595-1607.

Friedman K, Brewer B, Fangman W (1997) Replication profile of Saccharomyces cerevisiae chromosome VI. Genes Cells 2: 667-678.

Gerbi S (2004) Mapping origins of DNA replication in eukaryotes. Meth Mol Biol 296: 167-180.

Gilbert D (2001) Making sense of eukaryotic DNA replication origins. Science 294: 96-100.

Gilbert D (2002) Replication timing and metazoan evolution. Nat Genet 32: 336-337.

Gilbert D (2004) Timeline: In search of the holy replicator. Nat Rev Mol Cell Biol 5: 848-855.

Goldman M, Holmquist G, Gray M, Caston L, Nag A (1984) Replication timing of genes and middle repetitive sequences. Science 224: 686-692.

Heinzel S, Krysan P, Tran C, Calos M (1991) Autonomous DNA replication in human cells is affected by the size and the source of the DNA. Mol Cell Biol 11: 2263-2272.

Hsiao C, Carbon J (1979) High-frequency transformation of yeast by plasmids containing the cloned yeast ARG4 gene. Proc Natl Acad Sci USA 76: 3829-3833.

Hyrien O, Maric C, Mechali M (1995) Transition in specification of embryonic metazoan DNA replication origins. Science 270: 994-997.

Jeon Y, Bekiranov S, Karnani N, et al. (2005) Temporal profile of replication of human chromosomes. Proc Natl Acad Sci USA (in press).

Katou Y, Kanoh Y, Bando M et al. (2003) S-phase checkpoint proteins Tof1 and Mrc1 form a stable replication-pausing complex. Nature 424: 1078-1083.

Khodursky A, Peter B, Schmid M et al. (2000) Analysis of topoisomerase function in bacterial replication fork movement: use of DNA microarrays. Proc Natl Acad Sci USA 97: 9419-9424.

Klevecz R, Bolen J, Forrest G, Murray D (2004) A genomewide oscillation in transcription gates DNA replication and cell cycle. Proc Natl Acad Sci USA 101: 1200-1205.

Lipford J, Bell S (2001) Nucleosomes positioned by ORC facilitate the initiation of DNA replication. Mol Cell 7: 21-30.

MacAlpine D, Rodriguez H, Bell S (2004) Coordination of replication and transcription along a Drosophila chromosome. Genes Dev 18: 3094-3105.

Mendez J, Stillman B (2003) Perpetuating the double helix: molecular machines at eukaryotic DNA replication origins. Bioessays 25: 1158-1167.

Meselson M, Stahl FW (1958) The replication of DNA in Escherichia coli. Proc Natl Acad Sci 44: 671-682.

Mostoslavsky R, Singh N, Tenzen T et al. (2001) Asynchronous replication and allelic exclusion in the immune system. Nature 414: 221-225.

Newlon C, Theis J (1993) The structure and function of yeast ARS elements. Curr Opin Genet Dev 3: 752-758.

Newlon C, Collins I, Dershowitz A et al. (1993) Analysis of replication origin function on chromosome III of
Saccharomyces cerevisiae. Cold Spring Harb Symp Quant Biol 58: 415-423.

Pasero P, Shimada K, Duncker B (2003) Multiple roles of replication forks in $\mathrm{S}$ phase checkpoints: sensors, effectors and targets. Cell Cycle 2: 568-572.

Raghuraman M, Winzeler E, Collingwood D et al. (2001) Replication dynamics of the yeast genome. Science 294: 115-121.

Rao H, Marahrens Y, Stillman B (1994) Functional conservation of multiple elements in yeast chromosomal replicators. Mol Cell Biol 14: 7643-7651.

Remus D, Beall E, Botchan M (2004) DNA topology, not DNA sequence, is a critical determinant for Drosophila ORC-DNA binding. EMBO J 23: 897-907.

Ren B, Robert F, Wyrick J et al. (2000) Genome-wide location and function of DNA binding proteins. Science 290: 2306-2309.

Reynolds A, McCarroll R, Newlon C, Fangman W (1989) Time of replication of ARS elements along yeast chromosome III. Mol Cell Biol 9: 4488-4494.

Santocanale C, Diffley J (1998) A Mec1- and Rad53-dependent checkpoint controls late-firing origins of DNA replication. Nature 395: 615-618.

Schübeler D, Scalzo D, Kooperberg C, van Steensel B, Delrow J, Groudine M (2002) Genome-wide DNA replication profile for Drosophila melanogaster: a link between transcription and replication timing. Nat Genet 32: 438-442.

Schübeler D, MacAlpine D, Scalzo D et al. (2004) The histone modification pattern of active genes revealed through genome-wide chromatin analysis of a higher eukaryote. Genes Dev 18: 1263-1271.

Segurado M, de Luis A, Antequera F (2003) Genome-wide distribution of DNA replication origins at Arich islands in Schizosaccharomyces pombe. EMBO Rep 4: 1048-1053.

Shirahige K, Hori Y, Shiraishi K et al. (1998) Regulation of DNA-replication origins during cell-cycle progression. Nature 395: 618-621.

Spellman P, Sherlock G, Zhang M et al. (1998) Comprehensive identification of cell cycle-regulated genes of the yeast Saccharomyces cerevisiae by microarray hybridization. Mol Biol Cell 9: 3273-3297.

Stambrook P, Flickinger R (1970) Changes in chromosomal DNA replication patterns in developing frog embryos. $J$ Exp Zool 174: 101-113.

Stinchcomb D, Struhl K, Davis R (1979) Isolation and characterisation of a yeast chromosomal replicator. Nature 282: $39-43$.

Taljanidisz J, Popowski J, Sarkar N (1989) Temporal order of gene replication in Chinese hamster ovary cells. Mol Cell Biol 9: 2881-2889.

Vashee S, Cvetic C, Lu W, Simancek P, Kelly T, Walter J (2003) Sequence-independent DNA binding and replication initiation by the human origin recognition complex. Genes Dev 17: 1894-1908.

Vogelauer M, Rubbi L, Lucas I, Brewer B, Grunstein M (2002) Histone acetylation regulates the time of replication origin firing. Mol Cell 10: 1223-1233.

White E, Emanuelsson O, Scalzo D et al. (2004) DNA replication-timing analysis of human chromosome 22 at high 
resolution and different developmental states. Proc Natl Acad Sci USA 101: 17771-17776.

Woodfine K, Fiegler H, Beare D et al. (2004) Replication timing of the human genome. Hum Mol Genet 13: 191-202.

Wyrick J,Aparicio J,Chen Tetal.(2001)Genome-widedistribution of ORC and MCM proteins in S. cerevisiae: high-resolution mapping of replication origins. Science 294: 2357-2360.
Yabuki N, Terashima H, Kitada K (2002) Mapping of early firing origins on a replication profile of budding yeast. Genes Cells 7: 781-789.

Zou Y, Gryaznov S, Shay J, Wright W, Cornforth M (2004) Asynchronous replication timing of telomeres at opposite arms of mammalian chromosomes. Proc Natl Acad Sci USA 101: $12928-12933$. 